\title{
Serological Negative Cytomegalovirus Pneumonia Complicated with Bronchiolitis Obliterans Organizing Pneumonia in an Immunocompetent Patient: A Case Report
}

\author{
Chunxia Chen, Wan Chen, Xia Liu, Li Li and Luying Huang
}

Department of Hyperbaric Oxygen, The People's Hospital of Guangxi Zhuang Autonomous Region, Nanning, Guangxi 530021, P.R. China

Corresponding author: Chunxia Chen, Department of Respiratory, The People's Hospital of Guangxi Zhuang Autonomous Region, 6 Taoyuan Road, Nanning 530021, Guangxi Zhuang Autonomous Region, P. R. China, Tel: +86 771 2186451; Fax: +86 7712802018; E-mail: 40192385@qq.com

Received: 04 December 2015; Accepted: 30 July 2016; Published: 02 August 2016

Citation: Chen C, Chen W, Liu X, et al. Serological Negative Cytomegalovirus Pneumonia Complicated with Bronchiolitis Obliterans Organizing Pneumonia in an Immunocompetent Patient: A Case Report. Ann Clin Lab. 2016, 4: 3.

\section{Abstract}

A 63 year-old man presented with fever, cough, sputum and tachypnea. The latter deteriorated rapidly. A thoracic computed tomography (CT) scan revealed bilateral reticular shadows appearance and quickly spread around two lungs. Notably, CMV PCR was also negative. However, based on inclusion bodies found in lung tissue transbronchial biopsy (TBLB), the diagnosis of CMV pneumonia was confirmed. Lung biopsy showed bronchiolitis obliterans organizing pneumonia (BOOP). Following ganciclovir and steroid treatment, he made a gradually recovery and was discharged seven days later. This case demonstrated that CMV pneumonia may associate with bronchiolitis obliterans organizing pneumonia in an immunocompetent patient.

Keywords: Cytomegalovirus pneumonia; Bronchiolitis obliterans organizing pneumonia; Immunocompetent

\section{Introduction}

The clinical manifestations of cytomegalovirus (CMV) infection may be divided into acute primary infection and reactivation. Primary CMV infection occurs most frequently during breastfeeding and childhood [1], and is asymptomatic or present as a mild mononucleosis-like syndrome in immunocompetent adults [2]. Then the virus persists for life in the host, generally in the latent stage. Reactivation of CMV from latency is well known in immunocompromised patients [3], and it can affect almost any organ. However, severe CMV pneumonia is rare in immunocompetent hosts [4-6]. Presently, the precise molecular and immunological mechanisms involved are still unknown.

Bronchiolitis obliterans organizing pneumonia (BOOP) is a clinical condition characterized by cough, malaise, fever, and dyspnoea and no response to antibiotics [7]. Its chest radiograph described as local, multiple and diffuses changes [8]. It may be found with allergic alveolitis, irradiation pneumonitis, drug reactions and other organizing infections $[9,10]$. Cases involved in viral etiology complicated with BOOP have sporadically been reported. Here, we present a case of CMV pneumonia associated with BOOP in an immunocompetent patient by therapy of ganciclovir, which is the first such case ever reported as far as we known.

\section{Case Report}

A 63-year-old man was referred to our hospital due to a 10 day history of cough and fever. He had a paroxysmal cough with yellow sputum. Fever, with wheezing mainly appeared in the afternoon and night and the highest temperature was 39.3(102.74). His earlier medical history included hypertension, diabetes, and gout. His previous family history was unremarkable. His vital signs were as follows: body temperature: 36.5 (97.7), heart rate: 98 beat per minute (BPM), blood pressure: $98 / 61 \mathrm{mmHg}$.

On physical examination, late inspiratory crackles were noted in the bilateral lower lung fields. White blood cells (WBC) count was $14.2 \times 109 / \mathrm{L}$, with $81.9 \%$ neutrophils (NEUTR), $12.8 \%$ lymphocytes (LYR) and $0.53 \times 109 / \mathrm{L}$ monocytes (MONOR) (Figure 1). Serum C-reactive protein (CRP) level was $110.67 \mathrm{mg} / \mathrm{L}$.

Arterial partial pressure of oxygen $\left(\mathrm{PaO}_{2}\right)$ was $75.5 \mathrm{mmHg}$, with $25.8 \mathrm{mmHg}$ arterial partial pressure of $\mathrm{CO}_{2}\left(\mathrm{PaCO}_{2}\right)$ and $94.7 \%$ oxygen saturation $\left(\mathrm{SaO}_{2}, 2 \mathrm{~L} / \mathrm{min}\right.$ oxygen supply via nasal cannula). Because of the fever and the signs of respiratory infections, we experientially started antibiotic treatment with piperacillin tazobactam. However, the patient's temperature did not reduce and shortness of breath developed in persistent 4 days of hospital admission.

Arterial blood gas measurements revealed failure of respiration $\left(\mathrm{PaO}_{2}: 53.5 \mathrm{mmHg}, \mathrm{PaCO}_{2}: 35.6 \mathrm{mmHg}, \mathrm{SaO}_{2}\right.$ : $87.7 \%)$ with counterbalanced metabolic acidosis $\left(\mathrm{HCO}_{3}{ }^{-}: 21\right.$ $\mathrm{mmol} / \mathrm{l}, \mathrm{pH}$ 7.39) when using $2 \mathrm{~L} / \mathrm{min}$ oxygen by nasal cannula. Laboratory investigation results were as follows: mycoplasma pneumonia antibody: 1:40 (positive), bacterial endotoxin (the total content of lipopolysaccharide of gram negative bacteria): 
$49.14 \mathrm{pg} / \mathrm{mL}$, plasma fibrinogen (Fg): $7.51 \mathrm{~g} / \mathrm{L}$, activated partial thromboplastin time (APTT): 45.9 second.

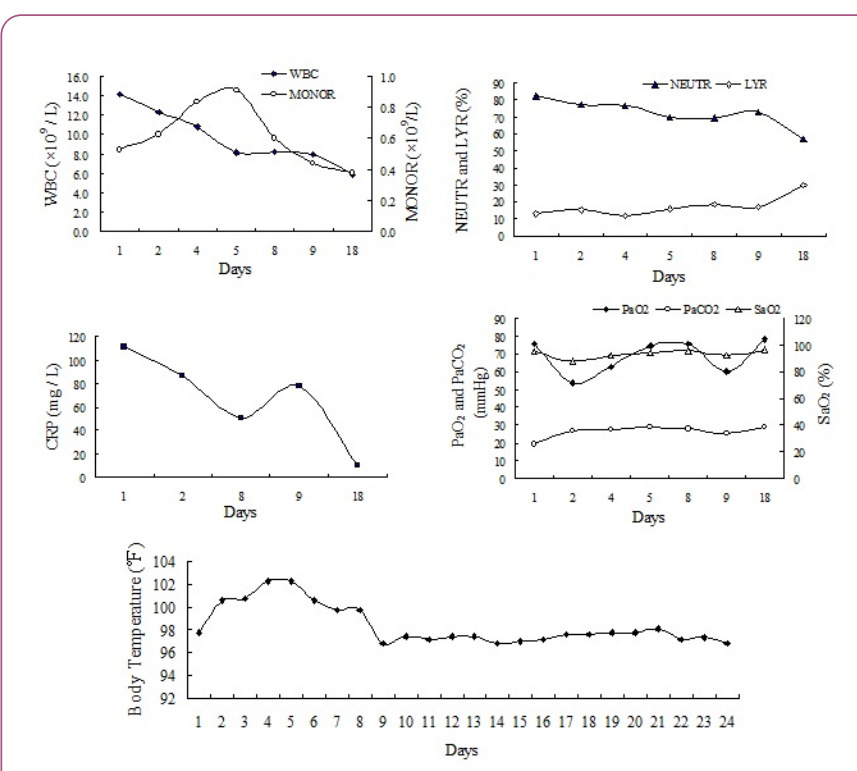

Figure 1 Time-course of the laboratory tests of blood routine, arterial blood gas analysis and body temperature.

The electrocardiogram showed sinus tachycardia with T wave change. Chest $X$-ray found two pulmonary infections, not except pulmonary interstitial lesions (Figure 2A). A thoracic computed tomography (CT) scan revealed pulmonary interstitial lesions (shadow of grid in both lungs), and mediastinal lymph node enlargement (Figure 2D). Then, 3.5 L/min oxygen was supplied with face mask and antibiotic treatment was switched to Cefoperazone Sodium and Sulbactam Sodium plus Levofloxacin.

Considering diagnosis of an interstitial pneumonia, relevant laboratory tests and fiberoptic bronchoscopy were performed on day 5. Laboratory test results were as follows: CMV PCR: negative, CMV IgM: negative, immunoglobulin IgG: positive, HIV: negative, WBC: $7.96 \times 109$ /L, NEUTR: $72.4 \%$, LYR: $17 \%$, CRP: $77.51 \mathrm{mg} / \mathrm{L}$, immunologic function ( $\mathrm{T}, \mathrm{B}, \mathrm{NK}$ cells): normal, T-lymphocyte subsets: normal. Markers for autoimmune diseases including perinuclear-ANCA ( $p-A N C A)$, cytoplasmic-ANCA (c-ANCA), ANA and anti-dsDNA were all negative.

Pulmonary function suggested moderate restrictive ventilatory dysfunction. $\mathrm{X}$-ray and $\mathrm{CT}$ scan revealed lesions on upper pulmonary were absorbed, but it was exacerbated in the right lower lung (Figure 2B and 2E).

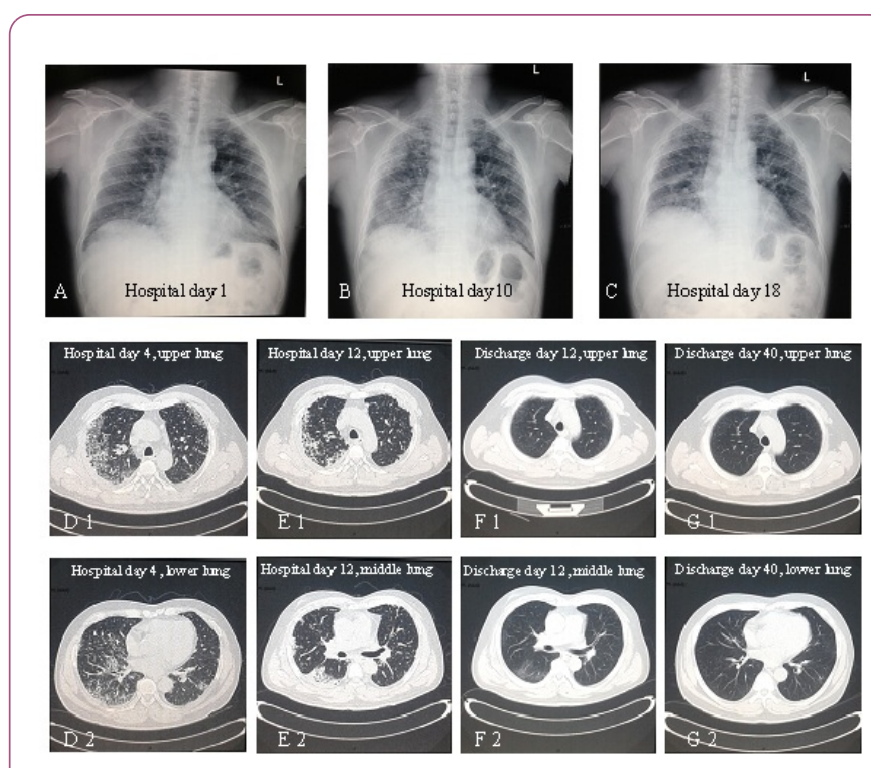

Figure 2 Radiological changes including chest $X$-ray $(A, B$, and $C$ ) and $C T$ scan (D, E, F, and $G)$. Bilateral reticular shadows in both lung fields (A, D1, D2) were showed in radiological test. Reticular shadows quickly spread around two lungs ( $B, E 1, E 2)$. Lesions in both lungs regressed significantly following ganciclovir (C, F1, F2).

A bronchoscopy showed bilateral bronchitis (Figure 3A). Bronchoalveolar lavage (BAL) fluid was negative for mycobacteria and PAS stain. Differential cell count showed $11 \%$ neutrophils, $19 \%$ lymphocytes and $70 \%$ macrophages. Intranuclear and intracytoplasmic inclusion bodies were found in lateral basal segment of the lung tissue transbronchial biopsy (TBLB) of the right lower (Figure 3B). Based on the diagnosis of cytomegalovirus pneumonia with organization, treatment with ganciclovir $(5 \mathrm{mg} / \mathrm{kg}$ ) via intravenous infusion every 12 hours was commenced on day 8. According to above finding combining with clinical symptoms (fever disappear), the antibiotic treatment was changed to Moxifloxacin on day 8. After 1 week of antivirus therapy, cough persistent. He underwent lung biopsy via CT-guided. A lung biopsy revealed BOOP (Figure $\mathbf{3 C}$ ). Then the patient treat with oral steroid hormone. Nevertheless his symptoms gradually improved in the following 1 week. Chest $\mathrm{X}$-ray revealed that two lung lesions were better than before (Figure $\mathbf{2 C}$ ).

Treatment was changed to oral ganciclovir, $0.6 \mathrm{~g} / \mathrm{d}$ (q12h) for 7 days, discharge. In his follow-up, shadow of grid in both lungs regressed significantly (Figure $\mathbf{2 F}$ and $\mathbf{2 G}$ ). He is still under follow-up at the outpatient clinic. 

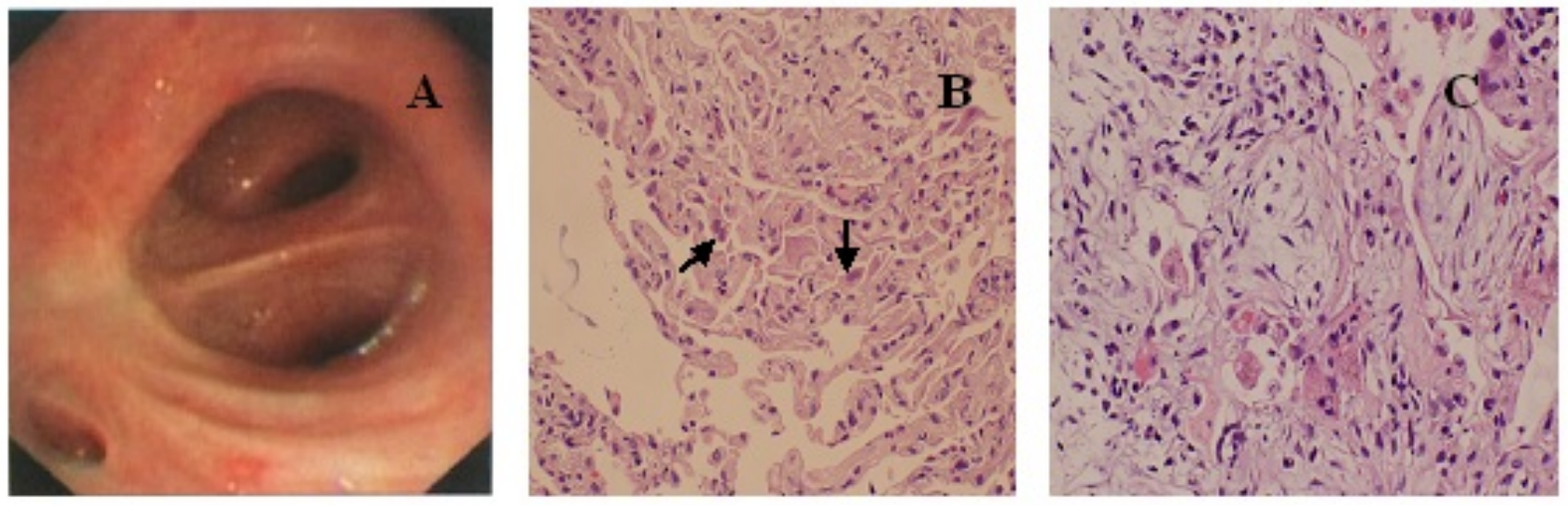

Figure 3 Endoscopic finding $(A)$ and microscopic views of histologic specimen (B, C). A, Chronic inflammation was appeared in the mucosa of bronchus. $B$, intranuclear and intracytoplasmic inclusion bodies were found in Lateral basal segment of the lung tissue.

\section{Discussion}

CMV is a DNA virus in the family Herpesviridae, and its infection is common. Indeed, $40 \%$ to $100 \%$ of adults are infected with CMV all over the world [11,12]. CMV infections in the general population are usually subclinical. The symptomatic CMV infection mainly occurred in immunocompromised patients with immune dysfunction, including patients with AIDS, malignancy, transplant recipients, and neonates $[2,13]$. In cases with immunodeficiency, CMV infection can cause pneumonia, retinitis, hepatitis, encephalitis, demyelinating polyneuropathy, myocarditis, pancreatitis, gastrointestinal diseases and adrenal diseases, and so on $[13,14]$. On the contrary, severe life-threatening infection induced by $\mathrm{CMV}$, including CMV pneumonia, is handful in immunocompetent individuals [4-6]. In this paper we describe a case of CMV pneumonia, associated with BOOP in an immunocompetent host, and effectively treated with ganciclovir.

CMV pneumonia often presents as severe viral pneumonia [6], with diffuse interstitial infiltrates on chest $X$-ray and respiratory failure [5]. The laboratory diagnosis may be difficult, and are made serologically or pathologically. Generally, the serologic assays to detect human CMV infection are including CMV antibodies (IgM,IgG) titer, CMV protein pp65, and CMV PCR $[15,16]$. Importantly, histopathological detection of CMV inclusion bodies on transbronchial or open lung biopsy is pathognomonic, since CMV pneumonia in immunocompetent patients represents a rare event [5].

Our patient presented with non-specific symptoms (cough and fever), which had developed over the course of 10 days. The symptoms were deteriorated after 2 days in hospital (severe hypoxaemia), despite empirical antibiotic therapy. The diagnosis of CMV pneumonia was based on TBLB, which was found intranuclear and intracytoplasmic inclusion bodies in giant cells as the gold standard. Moreover, serum CMV IgG antibody positive was another diagnostic index. The radiological picture including chest X-ray and $\mathrm{CT}$ scan showing bilateral reticular shadows in both lung fields supported the diagnosis of viral pneumonia. In our case, mononucleosis of blood routine was also observed (Figure 1). However, serum IgM CMV was negative in this case, which was in accordance with Eddleston report [4]. CMV PCR was also negative. It may be explain by false negative results induced by transient nature of the viremia [17].

During the hospitalization, his lymphocytes rate decreased significantly. Lymphopenia was caused by CMV infection for both direct inhibition of progenitor cell growth and the failure of stem cell self-renewal [18]. It may be noted that the immune function analysis such as T cell subset, B cell, and NK cell, analysis were normal. Transient or subclinical $T$ cell abnormality and an inversion of the CD4/CD8 T-cell ratio were not observed in this case. In HIV disease, a decrease in CD4+ cells $<100 \times 106 / \mathrm{L}$ representing a high risk for CMV disease [19]. Furthermore, our patient exhibited no evidence of other immunodeficiencies (HIV: negative) and autoimmune diseases (autoimmune antibody: negative).

BOOP was first described by Epler et al. [20] and since then has been extensively investigated and reported throughout the world [21]. There are many infectious agents may be associated with BOOP, such as cytomegalovirus (CMV), herpes simplex virus (HSV) [22]. Parasitic and pneumocystis carinii infections has also been reported $[23,24]$. Because BOOP represent way in which the lung may respond to an inflammatory stimulus, the key pathophysiological findings are related to the inflammatory pathway. Histological features include granulation tissue in the walls of the alveoli and bronchioles are significant, associated with infiltration in interstitial and tracheal cavity by different degrees of mononuclear cells and foam cells. Type II alveolar epithelial cell hyperplasia may also be seen $[20,25]$. In the current patient, BOOP was diagnosed in lung biopsy one week later than TBLB, suggesting CMV may be the most probable pathogen.

Although there is no recommended standard duration for ganciclovir treatment for patients with CMV pneumonia, the medication could only be started after definitive diagnosis and 
is commonly administered for two to three weeks. As soon as treatment with ganciclovir, the clinical condition of our patient was improved, with complete resolution of the clinical and radiological picture under one month follow-up.

\section{Conclusions}

We report an acute $\mathrm{CMV}$ infection case, which presented with fever, cough, sputum and tachypnea. The latter deteriorated rapidly and is associated with respiratory failure. CT scan revealed a little change of ground glass on onset, but the change quickly spread around two lungs. Following of pathologic examination (TBLB), the diagnosis of CMV pneumonia was confirmed. For patients with no immunodeficiency disorders, it is very easy to misdiagnosis. The current report suggests that when chest CT showing interstitial lesions, in addition to consider tuberculosis, bacterial pneumonia and other viral diseases, the CMV pneumonia should be included in elderly patients with a variety of basic disease. Importantly, this case demonstrated that cytomegalovirus pneumonia may associate with BOOP in an immunocompetent patient. Ganciclovir treatment is effective. Further studies are necessary to analyze the relationships and its underlying mechanism between the CMV pneumonia and BOOP.

\section{Author's contributions}

CXC drafted and wrote the manuscript. WC, LL and LYH took care of the patient and contributed to coordinating the manuscript. XL carried out the microbiological assays. LYH contributed to the draft of the manuscript and revised the manuscript. All authors have read the manuscript and approved its final version.

\section{Acknowledgments}

We are grateful to Prof. Zhiqiang Qin for his theoretical supports.

\section{References}

1. Hamprecht K, Maschmann J, Vochem M, Dietz K, Speer CP, et al. (2001) Epidemiology of transmission of cytomegalovirus from mother to preterm infant by breastfeeding. Lancet 357: 513-518.

2. Carter D, Olchovsky D, Pokroy R, Ezra D (2006) Cytomegalovirusassociated colitis causing diarrhea in an immunocompetent patient. World J Gastroenterol 12: 6898-6899.

3. Kralickova P, Mala E, Vokurkova D, Krcmova I, Pliskova L, et al. (2014)Cytomegalovirus Disease in Patients with Common Variable Immunodeficiency: Three Case Reports. International Archives of Allergy and Immunology 163: 69-74.

4. Eddleston M, Peacock S, Juniper M, Warrell DA (1997) Severe cytomegalovirus infection in immunocompetent patients. Clin Infect Dis 24: 52-56.
5. Cunha BA (2010) Cytomegalovirus pneumonia: communityacquired pneumonia in immunocompetent hosts. Infect Dis Clin North Am 24: 147-158.

6. Cohen JI, Corey GR (1991) Cytomegalovirus infection in the normal host. Medicine 1985; 64: 100-114.

7. Geddes DM. BOOP and COP. Thorax 46: 545-547.

8. Hansell DM (1992) What are bronchiolitis obliterans organizing pneumonia (BOOP) and cryptogenic organizing pneumonia (COP)? Clin Radiol 45: 369-70.

9. Cunha BA, Syed U, Mickail N (2012) Renal transplant with bronchiolitis obliterans organizing pneumonia (BOOP) attributable to tacrolimus and herpes simplex virus (HSV) pneumonia. Heart \& Lung 41: 310-315.

10. Bitzan M, Ouahed JD, Carpineta L, Bernard C, Bell LE (2010) Cryptogenic organizing pneumonia after rituximab therapy for presumed post-kidney transplant lymphoproliferative disease. Pediatric Nephrology 25: 1163-1167.

11. de la Hoz RE, Stephens G, Sherlock C (2002) Diagnosis and treatment approaches of CMV infections in adult patients. J Clin Virol 25: 1-12.

12. Sanadgol N, Ramroodi N, Ahmadi GA, Komijani M, Moghtaderi A, et al. (2011) Prevalence of cytomegalovirus infection and its role in total immunoglobulin pattern in Iranian patients with different subtypes of multiple sclerosis. New Microbiologica 34: 263-274.

13. Chiotan C, Radu L, Serban R, Cornacel C, Cioboata M, et al. (2014) Cytomegalovirus retinitis in HIV/AIDS patients. Journal of medicine and life 7: 237-240.

14. Liu WL, Lai CC, Ko WC, Chen YH, Tang HJ, et al. (2011) Clinical and microbiological characteristics of infections caused by various Nocardia species in Taiwan: a multicenter study from 1998 to 2010. Eur J Clin Microbiol Infect Dis 30: 1341-1347.

15. Beam E, Razonable RR (2012) Cytomegalovirus in solid organ transplantation: epidemiology, prevention, and treatment. Curr Infect Dis Rep 14: 633-641.

16. Westman $\mathrm{G}$, Berglund D, Widen J, Ingelsson M, Korsgren $\mathrm{O}$, et al. (2014) Increased Inflammatory Response in Cytomegalovirus Seropositive Patients with Alzheimer's Disease. PloS one 9: 96779.

17. Carney WP, Rubin RH, Hoffman RA, Hansen WP, Healey $K$, et al. (1981) Analysis of T lymphocyte subsets in cytomegalovirus mononucleosis. J Immunol 126: 2114-2116.

18. Boeckh $M$, Leisenring $W$, Riddell SR, Bowden RA, Huang ML, et al. (2003) Late cytomegalovirus disease and mortality in recipients of allogeneic hematopoietic stem cell transplants: importance of viral load and T-cell immunity. Blood 101: 407-414.

19. Gunthard HF, Aberg JA, Eron JJ, Hoy JF, Telenti A, et al. (2014) Antiretroviral treatment of adult HIV infection: 2014 recommendations of the International Antiviral Society-USA Panel. JAMA 312: 410-425.

20. Epler GR, Colby TV, McLoud TC, Carrington CB, Gaensler EA (1985) Bronchiolitis obliterans organizing pneumonia. N Engl J Med 312: 152-158.

21. Kute VB, Patel MP, Patil SB, Shah PR, Vanikar AV, et al. (2013) Bronchiolitis obliterans organizing pneumonia (BOOP) after renal transplantation. Int Urol Nephrol 45: 1517-1521. 
22. White KA, Ruth-Sahd LA (2007) Bronchiolitis obliterans organizing pneumonia. Crit Care Nurse 27:53-66.

23. Yale SH, Adlakha A, Sebo TJ, Ryu JH (1993) Bronchiolitis obliterans organizing pneumonia caused by Plasmodium vivax malaria. Chest 104: 1294-1296.

24. Kleindienst R, Fend F, Prior C, Margreiter R, Vogel W (1999) Bronchiolitis obliterans organizing pneumonia associated with
Pneumocystis carinii infection in a liver transplant patient receiving tacrolimus. Clin Transplant 13: 65-67.

25. Moore SL (2003) Bronchiolitis obliterans organizing pneumonia: a late complication of stem cell transplantation. Clin J Oncol Nurs 7: 659-662. 